\title{
Editorial
}

\section{TENDENCIAS DE LA EDUCACIÓN SUPERIOR}

EN AMÉRICA LATINA Y EL CARIBE

P arala Conferencia Regional deEducación Superior (CRES), que se llevó a cabo del 4 al 6 de junio de este año, Unesco y el Instituto Internacional para la Educación Superior en América Latina y el Caribe (IESALC) presentaron una interesante obra titulada Tendencias de la Educación Superior en América Latina y el Caribe ${ }^{1}$, donde agrupan los temas de mayor actualidad, mostrando un panorama amplio de lo que acontece en este campo en la región. Es un texto básico para todos aquellos que están comprometidos con la universidad y con las políticas educativas.

La obra es el resultado del proyecto presentado por Axel Didriksson ante el IESALC - Unesco, en el 2006, orientado a "identificar los escenarios posibles ylas tendencias de largo plazo en la educación superior en la región, desde una visión prospectiva"2. Está basada "en el principio de que la educación es un bien público y un derecho social y universal, y en la convicción de que ella tiene un papel estratégico que jugar en los procesos de desarrollo sustentable de los países de la región"”.

Dentro de las tendencias que se identifican en la obra figuran la transición de una situa- ción en donde primaban las universidades públicas a un sistema "complejo, heterogéneo y segmentado socialmente"t. De instituciones con un solo campus urbano, se ha derivado a macrouniversidades nacionales, con multicampus y una cantidad interesante de ofertas, muy diversificadas.

Así, las pocas instituciones privadas que existían han aumentado de manera notoria, generando un conjunto variado, en el que está presente la iniciativa particular de manera dominante. Además de escuelas técnicas y vocacionales de nivel medio superior, se ha llegado a instituciones politécnicas y tecnológicas de niveles medio, medio superior y superior.

La limitada investigación científica, realizada por un número reducido de investigadores, que caracterizaba a los países de la región, se ha multiplicado de manera apreciable. Hoy se cuenta con una diversidad de laboratorios y de institutos de ciencias que trabajan en todas las áreas del pensamiento humano y que avanzan, pese a sus limitaciones.

Así mismo, el número de estudiantes que tenían acceso a la educación superior ha sido

1. Tendencias de la Educación Superior en América Latina y el Caribe ha sido editada por IESALC-UNESCO, 2008. Se encuentra disponible en el sitio Web www.iesalc.unesco.org.ve

2. Tendencias de la Educación Superior en América Latina y el Caribe. Ana Lucía Gazzola y Axel Didriksson. Prefacio, IESALC-UNESCO, 2008, Pág. 1.

3. Ídem, pág. 3 .

4. Ídem, Capítulo I, "Contexto global y regional de la educación superior en América Latina y el Caribe", Axel Didriksson (con la colaboración de Efraín Medina, Miguel Rojas Mix, Lincoln Bizzozero y Javier Pablo Hermo), pág. 2. 
ampliado prodigiosamente, a tal punto, que se puede decir que "la región vive la masificación de la demanda social por la educación superior" 5 .

Es un interesante momento de cambio, caracterizado por la presencia de nuevos conocimientos y tecnologías, especialmente en informática, telecomunicaciones, biotecnología y nanotecnología, que abren otros horizontes, a los que están vinculadas de manera decisiva las instituciones de educación superior.

Pero estas tendencias de cambio implican, a la vez, grandes desafíos. La educación superior deberá enfrentar procesos como la tendencia a la mercantilización de las universidades privadas o el impacto de las tecnologías que redefinen los ambientes de aprendizaje. Por otra parte, aparecen áreas de conocimiento basadas en novedosas formulaciones interdisciplinarias, que están sustituyendo la oferta de de las carreras tradicionales.

En otro sentido, la disminución de los recursos de los gobiernos dedicados a la educación superior y la presencia de nuevos sistemas de evaluación y acreditación que valoran el desempeño de las instituciones, de los programas y de las mismas personas que trabajan en ellas, hacen parte de los retos que será necesario encarar.

Finalmente, hay que mencionar la tendencia a la internacionalización de los procesos de aprendizaje y de conocimiento, la presencia activa de redes y asociaciones académicas, la movilidad de los estudiantes, y la evolución de los sistemas de transferencia de conocimientos y tecnologías.

El documento profundiza en estos temas de manera amplia y responsable, buscando favorecer las transformaciones que requieren las universidades para orientar la construcción de sociedades de conocimiento y promover políticas de Estado que garanticen el desarrollo de sus países.

El Capítulo III $^{6}$ de la obra aborda un tema de la mayor importancia. Se trata de la calidad, la pertinencia y la responsabilidad social de la universidad. Los autores establecen que no solamente todas las personas tienen el derecho a la educación, sino que el Estado debe de proveerla con calidad. Además, la educación no es un bien negociable, aunque esté impartida por actores privados.

Hay un punto clave en esta formulación y es el concepto de calidad. Sostienen los autores que es difícil definir esta palabra aplicada al complejo universo de la educación superior. Se habla mucho de ella, sin una operacionalización precisa de su significado. Esto se hace más complejo en el proceso de expansión y privatización que vive la región.

Anotan los autores que existe una noción restrictiva en torno a la educación que sustituye el desarrollo humano por un concepto economicista, que tiene gran importancia en los países ricos. Se supone que ciertas instituciones poseen un nivel alto de calidad porque son exclusivas y llegan a un número muy selecto de personas. Empero, es necesario dar valor a los aspectos locales. Si la educación es un bien público que beneficia a todos y que disminuye las desigualdades, su extensión a la gran mayoría de los ciudadanos debe significar un incremento y no una pérdida en su calidad, sobre todo en Latinoamérica y el Caribe.

5. Ídem, Capítulo I, pág. 1.

6. Capítulo III, "Calidad, pertinencia y responsabilidad social de la universidad latinoamericana y caribeña”. José Días Sobrinho (con la colaboración de Adolfo Stubrin, Elvira Martín, Luís Eduardo González y Oscar Espinoza, Pedro Goergen). 
Los autores concluyen que los procesos de evaluación y acreditación en la región deben partir de un concepto de calidad que se base en la premisa de la educación como bien público y que tenga en cuenta la pertinencia, la responsabilidad y el compromiso con la construcción de nacionalidad; la autonomía y la libertad de pensamiento; la internacionalización y la cooperación entre los Estados; el respeto y la confianza entre las agencias y los actores universitarios; el respeto al medio ambiente, y el cumplimiento de la misión institucional.

El Politécnico Grancolombiano, Institución Universitaria, (PG), avanza en el proceso de ampliar su respuesta a los desafíos identificados por Unesco-IESALC. Luego de ingresar a la red de Whitney internacional University System (WIUS), el número de estudiantes de programas formales ha crecido significativamente: de 7.138 en 2006-2 a 9.252 en el 2008-2.

La actividad investigativa contó también con un incremento apreciable: de 10 proyectos de investigación aplicada en 2007-2, desarrollados por 10 grupos de investigación, se pasó a 22 proyectos en el 2008-2, adelantados por 9 grupos registrados en Colciencias.

De acuerdo con WIUS, el PG ha realizado una importante reforma institucional. Los programas se agruparon en cuatro facultades fortalecidas y enfocadas al desarrollo académico: 1) Ciencias Administrativas Económicas; 2) Ciencias de la Comunicación y Artes; 3) Ingenierías y Ciencias Básicas, y 4) Ciencias Sociales.

Las facultades cuentan con el respaldo de doce departamentos académicos: 1) Administración y Contabilidad; 2) Comunicación; 3) Humanidades; 4)
Industrial; 5) Medios Audiovisuales; 6) Social; 7) Artes; 8) Economía y Finanzas; 9) Lenguas;

10) Sistemas y Matemáticas; 11) Mercadeo, y 12) Turismo.

El WIUS y el PG han lanzado el revolucionario sistema de Educación Distribuida, que consiste en una combinación de metodologías de la educación presencial y la educación a distancia.

La primera promoción del modelo Whitney deEducación Distribuida cuenta con más de 400 estudiantes en el campus principal y en tres sedes asociadas en Suba, Barranquilla y Anapoima. Así, la Educación Distribuida se convierte en un instrumento positivo para llevar la cobertura de la educación superior a un mayor número de colombianos. Por ello, el Gobierno Nacional ha mostrado su entusiasta con este Modelo.

Tendencias de la Educación Superior en América Latina y el Caribe es un primer documento de conjunto que promueve el debate sobre lo que debe ser la educación superior en el continente. Existe una presencia marcada de los Estados en la definición de los parámetros que se deben lograr. Sin embargo, es importante vincular al proceso de planeación la experiencia de las universidades, como el PG, así como de las personas que trabajan en ellas. Es conveniente, además, que se dé valor a los factores locales de cada uno de los países del continente. "La alianza entre calidad y pertinencia rechaza una eventual vinculación a ideas mercantiles y a lógicas empresariales, correspondiendo a una concepción de educación como bien público al servicio de la construcción de sociedades democráticas y justas en pro de la afirmación de la identidad nacional"7.

7. Ídem, Capítulo III, pág. 6. 